\title{
L'électrification des chemins de fer du Maroc
}

Dans une communication à la Société des Ingénieurs cipils, parue dans le Bulletin de mai-juin 1928 de celte Société, notre éminent confrère et ami, M. Parodi, dont on sait le rôle de toul premier plan tant pour l'électrifica ion des chemins de fer de Paris à Orléans, que pour celle des chemins de jer du Maroc, a consacré de nombreuses pages, remplies de chiffres, de documents statistiques et aussi d'observations personnelles du plus haut intèrêt, à cette question de l'électrification des chemins de fer du Protectorat.

Nous aurions mauvaise grâce à insister davantage sur la valeur de ce travail, facile à préjuger du mérite de l'auteur. Nous y renverrons le lecteur, mais il nous a semblé qu'il n'était pas sans intérêt, nous inspirant des trapaux de notre collìgue, de donner ci-après un aperçu de cette ounn grandiose. La source unique à laquelle nous venons de puiser, nous venons de l'indiquer plus haul, quant à notre prêteur, il est, du reste, des plus riches, ct ce n'est qu'à ceux-là, comme le veut le proverbe "qu'il $y$ a intérêt à emprunter."

\section{A. - Développement Économgue du Maroc. Réseau routier ET RÉSEAU FERRÉ}

On sait le développement extrêmement rapide de la consommation d'énergie électrique au Maroc. Cette consommation croît nait, en 1926 , environ 3.000 kilomètres, et 450 ont été achevés en 1927.

Même poussée, en ce qui concerne les chemins de fer quị comprennent 1.300 kilomètres à voie étroite, résultant surtout de

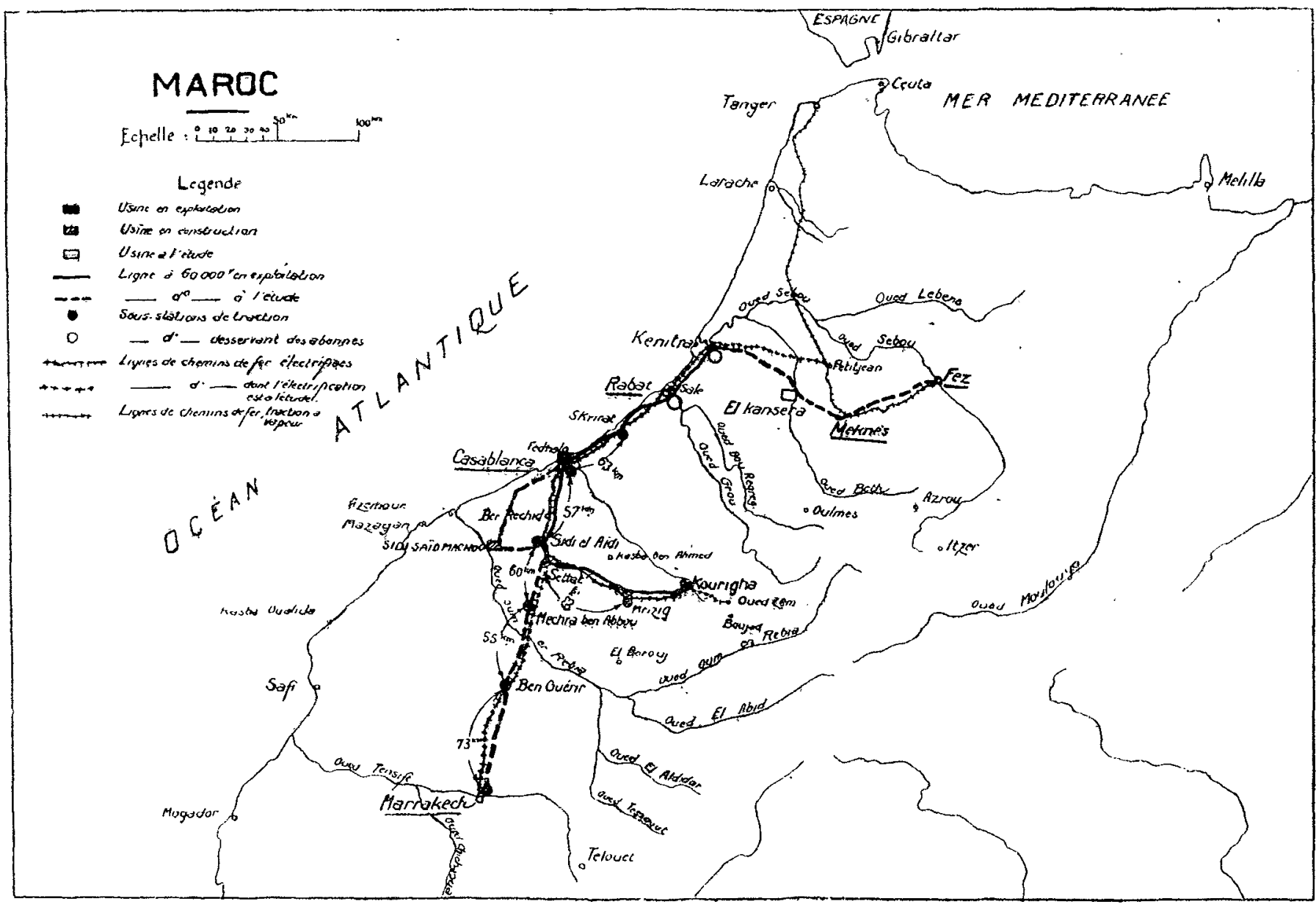

à la cadence de un million de Hwah par mois. Elle a dépassé 40 millions en 1928. Les chemins de fer électrifiés n'absorbent que le quart de celle-ci. Ceci semble faible, mais ce n'est qu'une aurore!

Le réseau routier du Maroc est extrêmement important. Il comprend notamment 3.400 kilomètres de routes principales et 1.650 kilomètres de routes secondaires.

Il est construit à l'allure de 200 kilomètres par an. Il compre- constructions de guerre et d'avant-guerre, et 800 kilomètres de voie normale en exploitation.

Les voies étroites sont des héritages d'avant-guerre et de guerre.

Les voies normales comprennent les deux réseaux du Tanget; Fez (Compagnie franco-espagnole), et des Chemins de fer du Maroc (Compagnie exclusivement française et constituée par ulr groupement qui comprend la Compagnie générale du Maroc le P.-L.-M., le P.-O. et la Compagnie marocaine. 
Réseau à voie étroite - Les six lignes du réseau à voie étroite en exploitation normale sont les suivantes :

\begin{tabular}{|c|c|}
\hline Oudja-Fez......... & $401 \mathrm{~km}$. \\
\hline Guereef-Tamdaleft............... & $215-$ \\
\hline Bir-Tam-Tam-Ahermoun ......... & $42-$ \\
\hline Kenitra-Ouezzan . ........ & $158-$ \\
\hline Ain-Defali-Fez-el-Bali . ............ & $64-$ \\
\hline Souk-el-Tleta-Mecha-el-Hadr ...... . & \\
\hline Rabat-Tiflet................... & $64-$ \\
\hline Casablanca-Marrakech............. & $286-$ \\
\hline
\end{tabular}

On a construit actuellement 209 kilomètres nouveaux à voie étroite, qui recevront le matériel disponible provenant des lignes à voic normale achevées.

\section{Réseau à voie normale (C. F. du Maroc) :}

Il comporte 1.018 kilomètres de voies, répartis en six lignes, qui sont les suivantes :

\begin{tabular}{|c|c|}
\hline 10 Kenitra-Petitjean (station du Tanger-Fez). & $85 \mathrm{~km}$. \\
\hline inca, par Rabat......... & \\
\hline $3^{\circ}$ Kenitra-Souk-el-Arba.......... & $80-$ \\
\hline $4^{\circ}$ Casablanca-Marrakech & $250-$ \\
\hline Sidi-el-Aïdi à Oued-Zemç. & 120 \\
\hline $\mathrm{Fe} z$ (frontière algérienne) & 355 \\
\hline
\end{tabular}

Ces lignes sont à voie unique avec possibilité de doublement, dès les premiers besoins.

Les lignes 1, 2, 5 (333 kilomètres exécutés) sont actuellement en service. La ligne 4, partiellement établie, comprend 73 kilomètres, Au total : 406. La traction est assurée électriquement sur 255 kilomètres, de Casablanca à Kourigha, depuis mars 1927, et de Casablanca à Salé, depuis décembre 1927. Sur le reste du parcours, la traction est assurée à la vapeur.

La ligne qui est la première électrifiée est celle de Kourigha, dite " des phosphates". Elle est exploitée, vu la pente continue depuis la mine jusqu'à Casablanca, avec la récupération. Le service est fait par des trains de 1.100 à 1.200 tonnes (descente en marche : 35 à 40 wagons, et remontant à vide 400 à 440 tonnes), d'où production presque complète de l'énergie nécessaire au train montant par le train descendant.

\section{B. - Production, transport, transformation et DISTRIBUTION DE L'ÉNERGIE}

Nous laissons de côté, comme en dehors de notre sujet, malgré son intérêt, la plupart des renseignements qui ont trait à la production et au transport de l'énergie, à sa distribution par lignes catenaires, aux locomotives et aux automotrices, ne retenant surtout que deux points : la constitution du matériel roulant, et les résultats de l'exploitation.

Usines. digues ef sous-stations. - Signalons cependant que l'energie électrique est actuellement produite par l'usine thermique de Casablanca (centrale de Roches-Noires), particulièrement bien placée, puịsqu'elle est à côté de l'Atlantique. Cette usine comprend trois turbo-alternatcurs pour $6.000 / 7.500$ kilowatts, donnant du triphasé à 50 périodes, 5.500 volts. On y adjoindra, et au besoin, on lui substituera l'usine hydro-électrique de Sidi-Machou (sur l'Oued-Oum-el-Rebia), à trentc-cinq kilomètres environ du débounché de ce fleuve sur l'Atlanticue, comportant quatre turbo-alternateurs à axe vertical de $5^{\mathrm{m} 50}$ de chute normale, avec puissance de 5.200 kilowatts pour $\cos \hat{q}$ $=0,8$ et sous 5.500 volts. De ces usines, partent des lignes à hautc tension (60.000 volts) longeant, en général, la voie du chemin de fer, sauf sur la section Casablanca-Sidi-Machou, la ligne joignant alors les usines thermiques et hydro-électriques. Les sous-stations en service sont au nombre de quatre : Casablanca $(2.000 \mathrm{KW})$, Sidi-el-Aïdi $(3.000 \mathrm{KW})$, Mrizig $(2.000 \mathrm{KW})$, avec quatre autres en projet ou en exécution. Elles sont desservies par les lignes à haute tension. La première seule n'est pas automatique (Casablanca). Les autres le sont, solution imposée surtout par l'insuffisance teclinique du personnel indigène.

Les groupes moteurs-générateurs de ces sous-stations (dcux ou trois par poste), sont constitués par des machines synchrones de 1.500 chevaux à 5.500 volts, 50 périodes, attelées à deux dynamos de 500 kilowatts, sous 1.500 volts associés en série. Les dynamos sont à pôles supplémentaires et en roulement de compensation. La technique des sous-stations, est visiblement inspirée, fait du reste nettement reconnu par leurs créateurs, de celle des sous-stations de la ligne américaine des Montagnes-Rocheuses.

A notre vif regret, nous ne pouvons entrer dans l'étude pourtant éminemment curieuse, du fonctionnement, en automatique, de ces sous-stations.

Lignes catenaires et circuit de retour. - Le circuit de traction est constitué par une ligne caténaire double, de deux fils de cuivre rainurés, étirés, de $107 \mathrm{~m} / \mathrm{m}^{2}$ de section, supportés, avcc interposition de pendules, par un câble porteur en acier, de $94 \frac{\mathrm{m} / \mathrm{m}^{2}}{\mathrm{~m}}$ de section.

Le circuit de retour comporte les deux files de rails éclissés électriquement ( $36 \mathrm{~kg}$. au mètre courant, coupons de 12 mètres), et un feeder de retour de $50^{\mathrm{m} / \mathrm{m}}{ }^{2}$ de section, monté sur le support à côté de la ligne caténaire. Ce feeder est destiné à assurer la continuité du circuit électrique de retour, de telle sorte que la différence de potentiel de 3.000 volts ne puisse s'établir entre deux coupons successifs de rails, si les deux voix venaient, au cours de travaux, à être déséclissées en même temps.

A signaler également les équipements des gares, un fecder auxiliaire reliant les voies principales en évitant les voies de service, et permettant, par la manœuvre d'un interrupteur spécial, d'isoler complètement ces gares sans interrompre la continuité du circuit général de traction.

\section{C. - Utilisation de l'énergie Électrique, et réscltats ÉCONOMIQUES OBTENUS EN MaTIËRE D'ÉLECTRIFICATION.}

Cette question est extrêmement complexe, et doit être étudiéc avec une grande prudence. M. Parodi, auteur principal de l'électrification du P.-O., a défini, dans de nombreuses publications, l'index économique d'exploitation.

L'expression ci-après :

$$
\varepsilon=\frac{a \mathrm{D}}{\mathrm{C}}
$$

dans laquelle a représente le loyer de l'argent, D le cours du dollar en francs, C le cours du charbon chargé sur lenders, les valeurs données pour la période 1920-1926, montrent que ce facteur varie de un à deux.

Il y a donc nécessité d'attendre que le temps ait fait son ouvre pour porter un jugement définitif, d'autant plus que l'électrification n'est que partielle, mème sur les réscatux les plus " à la page ", et, qu'en conséquence, les résultats obtenus sont "involontairument frelatés ». Tout au plus, pourrait-on déduire d'utiles renseignements de l'exploitation des chemins de fer fédéraux suisses, où l'électrification est beaucoup plus avancée. Cependant, c'est une opération en vase clos (usines spécialisées en courant monophasé, à 16,66 périodes), ce qui ne rend guère commode l'interprétation des résultats, bien que ceux-ci soient incontestablement favorables. En ce qui concerne le courant continu haute tension (1500 à 3.000 volts), nous n'avons comme 
exemples que le Midi français, le P.-O. et les chemins de fer du Maroc, auxquels il faut joindre la ligne américaine des Montagnes Rocheuses, dont les caractères spécifiques sont cependant: très différents.

Un point domine cependant la question : la parfaite sécurité assurée dans toutes les exploitations par la traction électrique. Les incidents y ont été nuls ou presque, quelques pannes seulement de transmission d'énergie, pannes inévitables, et liées à la mise en service des réseaux aussi complexes, aussi délicats, avec des méthodes d'exploitation inédites, et donc inexpérimentées.

M. Parodi, dans son étude, insiste sur deux points sur lesquels nous demandons la permission de nous arrêter avec lui, savoir : la variation de la consommation d'énergie avec le trafic, et la comparaison entre les résultats des calculs et ceux de l'exploitation. Il a étendu son étude au P.-O., aux chemins de fer du Maroc et à la ligne des Montagnes Rocheuses. Nous allons en donner ci-après le résumé.

\section{A. - Consommation d'énergie aux bornes des vachines (AUTOMOTRICES OU LOCONOTIVES)}

Actuellement, à des effets secondaires près, la consommation d'énergie des machines dépend simplement de leur service et non des autres unités en marche, comme pour les locomotives thermiques, du reste.

Consommation sur le P.-O. - Sur le P.-O. notamment, le calcul des énergies fournies et le relevé des énergies consommées, permet de déterminer, par tonne-kilomètre, les consommations suivantes :

\begin{tabular}{|c|c|c|c|}
\hline & $\begin{array}{l}\text { Valeurs caleuletes } \\
\text { (en wh) }\end{array}$ & $\begin{array}{l}\text { Valeurs mesuré.s } \\
\text { (en wh) }\end{array}$ & Erreurs commises \\
\hline Banlieue... . . . . . . . . & 29 & 27 & $+7 \%$ \\
\hline Omnibus et durects. & 21 & 21 & $0 \%$ \\
\hline Rapides et express ........... & 21 & 20 & $5 \%$ \\
\hline Messageries, denrées .......... & 21 & 21 & $0 \stackrel{0}{\circ}$ \\
\hline Marchandises.............. & $11, \tilde{5}$ & 11,6 & $1 \%$ \\
\hline
\end{tabular}

En outre de la concordance satisfaisante du calcul avec l'expérience, on notera la consommation relativement faible des trains de marchandises à la tonne-km. $30 \%$ de différence au moins, avec les trains de banlieue). Le motif en est que le coefficient de roulement des wagons de marchandises, est donné par la formule approximative ci-après, pour un véhicule de $P$ tonnes,

$$
\mathrm{R}=1,3+\frac{20}{\mathrm{P}}
$$

en $\mathrm{kg}$. par tonne. Les wagons vides ont donc un roulement plus pénible, à égalité de poids.

Les relevés globaux relatifs à Paris-Brétigny-Bourdan-Orléans, incorporent des valeurs moyennes, d'où une diminution de la consommation spécifique au fur et à mesure que s'emplacent sur le réseau des rapides, des express, des omnibus et des trains de messagerie, interposés avec les grands consommateurs que sont les trains de banlieue. Actuellement, on compte sur le P.-O. 16,7 watt-h. par tonne-km. remorquée, soit, sur un ensemble de 4 milliards $1 / 2$ de tonnes-km. remorquées par an.

Consommation sur les chemins de ler du Maroc. - Sur la ligne des phosphates, qui a été exploitée seule électriquement jusqu'en 1927, il est fait usage de la récupération. La ligne est en rampe continue de Casablanca $(+24 \mathrm{~m} 25)$ à Kourigha $\left(+792^{\mathrm{m}}\right)$. Les trains descendant pèsent de 720 à 800 tonnes. Les trains montants ne comportent que du matériel vide. Pour un train montant de 400 tonnes de matériel vide, la résistance pour roulement est de $3 \mathrm{~kg} .5$ par tonne. La dépense d'énergie est de 31,8 watt-heure par tonne-kilomètre.

Pour un train descendant de 1.100 tonnes environ de matéricl chargé, l'énergie récupéréc est d'environ 7 watts-heure par tonne- kilomètre totale, la résistance moyenne de roulement est de $2 \mathrm{~kg} .2$, la quantilé d'énergie employée par le démarrage est: d'environ 2,8 walts-heure par tonne-km. totale.

La formule de roulement du P.-O. :

$$
R=1, ;+\frac{20}{P}
$$

donnerait, pour un wagon vide (11 tonnes), la valeur de $3 \mathrm{~kg} .2$ par tonne, et pour les wagons pleins (29 à 30 tomnes), environ $2 \mathrm{~kg}$. La mise en service de nouveaux wagons permeltra d'améliorer ces conditions (wagons de 48 lonnes utiles.)

La consommation ressort donc, pour un train phosphatier, à 10,5 walts-heure par tomne-km. totale (parcours aller el retour). Pour un train normal (charge égale dans les deux sens), 20 watts-heure. Les trains phosphatiers sont au nombre de cing pour un train commercial. Donc, il ressort une consommation moyenne totale de 11,5 watls-h. par tonne-kilomètre totale, ou 12,7 watts-heure par lonne remorquée.

Le trafic hétérogène total correspond à 420 millions de lonnes$\mathrm{km}$. remorquées, soit 3 millions de tonnes-km. remorquées par kilomètre.

Consommation sur la ligne des Montagnes-Rocheuses. - Les renseignements sont nombreux, mais assez contradictoires. Il faut admettre, d'après M. Beeucokes (G.E.R., 1928), que la ligne en question, avec son profil en dents de scie, avec déclivité maximum de $20 \%$, entraîne une consommation de 16 watts-heure par tonne $\mathrm{km}$.

La consommation spécifique mesurée en haute tension, est, du reste, très variable avec le trafic. Cette détermination est, naturellement, assez délicate. Comparée avec ceux du P. -O. et des chemins de fer du Maroc, les résultats de la ligne des Montagnes-

\begin{tabular}{|c|c|c|c|}
\hline & M. R. & C. M. & P. $\mathrm{O}$. \\
\hline $\begin{array}{l}\text { Trafic comparé en millions de } \\
\text { tonnes-km., tonnes totales .... }\end{array}$ & 7,72 & 3,25 & 22,2 \\
\hline Tonnes remorqućes............. & 6,50 & 2,90 & 19,6 \\
\hline Nombre de sous stations ....... & 14 & 3 & \\
\hline Distance des sous-stations...... & $50 \mathrm{~km}$. & $45 \mathrm{~km}$. & $21 \mathrm{~km}$ \\
\hline $\begin{array}{l}\text { Consommation spécifique } p^{\mathrm{r}} \text { tonne } \\
\mathrm{km} \text {. totale et wh } \ldots \ldots \ldots \ldots \ldots\end{array}$ & 24,0 (1) & 20,95 (1) & 19,76 \\
\hline $\begin{array}{l}\text { Id., aux locomotives ........ } \\
\text { (1) Pour M. R., ce chiffre comprend } \\
\text { les services auxiliaires des loco- } \\
\text { motives. Pour C. M., il ne le } \\
\text { comprend pas. }\end{array}$ & 19,05 & 12,7 & 16,70 \\
\hline Rendement moyen... ....... & $67 \%$ & $55 \%$ & $75 \%$ \\
\hline
\end{tabular}
Rocheuses, ressortent les suivants, en millions de tonnes-km.:

On notera cependant que sur le P.-O. le rendement est mesuré à la haute tension, départ usines, et sur les C. M., aux bornes des sous-stations. Le rendement effectif du P. O . ainsi rectifié, s'élève à $84 \%$, auxquels il faut ajouter $1 \%$ (consommation des auxiliaires, chaufferettes, etc...), soit au total $85 \%$.

Sur le C. M., le rendement est de $55 \%$, auxquels il faut ajouter 2 à $3 \%$ pour les auxiliaires. Cette valeur est plus grande que pow le P.-O. et le M.-R., car ces machines sont à récupération et les auxiliaires dépensent dans les deux cas.

Quant aux M.-R. (rendement de $67 \%$ ) ce chiffre est un pell faible par rapport au P.-O., bien que la transmission d'énergie soit très simplifiée (cette énergie est livrée par des sociétés étrant gères sous la tension de transport aux chemins de fer). Ceci s'explique par ce fait que le trafic est 2,5 fois plus grand sur le P.-O. que sur le M.-R., et aussi du fait de l'emploi de commuta. trices à 1.500 volts au lieu de groupes générateurs à 3.000 volts Sur le C.-M., le trafic est trois fois plus faible que sur le M.-R. mais il montera. On pourra tomber à 18 watts-h. par tonne kni 
remorquée à haute tension, au lieu de 23,14, quand la quantité de phosphates transportée sera de l'ordre de 2,5 à 3 milliards de tonnes par an.

On notera cependant que les wagons américains du M.-R. (de très forts tonnages), sont donnés par les ingénieurs de cc pays comme ayant des résistances au roulement de 1,5 à $2 \mathrm{~kg}$. par tome. Or, les calculs faits avec les coefficients européens (formules du P.-O.) cadrent avec les chiffres relevés de l'énergie consommée sur le M.-R., d'où la conclusion qu'il y a beaucoup de wagons vides, ou en faible charge sur le M.-R.

\section{B. - Matériel locomoteur.}

Locomotives B.B. - Les chemins de fer du Maroc ont commandé dix locomotives B.B. à la " C.E.F. ». Chacune comporte deux bogies avec deux moteurs de traction à 1.500 volts, et une caisse unique contenant les appareils de manœuvre et de sécurité, ainsi ruc les machines auxiliaires (ventilateurs, excitatrices, etc...).

Constantes principales:
Longueur entre tampons . . . . . . . . . . .

Longueur de la caisse $\ldots \ldots \ldots \ldots \ldots \ldots \ldots \ldots$

Largeur de la caisse..................

Empallement d'un bogie...............

Ecartement normal des pivots d'un bogie....

Diamètre de la roue motrice .............

Epaisseur des bandages neufs ...........

Poids total de la locomotive.............

Poids total de la caisse équipée...........

Poids d'un bogie en ordre de marche........

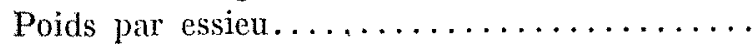

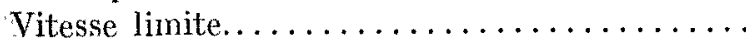

Puissance de la locomotive $\left\{\begin{array}{l}\text { continue......... } \\ \text { unihoraire....... }\end{array}\right.$
$11 \mathrm{~m} 870$

10743

2960

2800

5550

1400

$\begin{array}{lll}0 & 075\end{array}$

73 tonnes

33 -

$20-$

18 t. 250

$60 \mathrm{~km} . / \mathrm{h}$.

$1.050 \mathrm{ch}$. $1.340 \mathrm{ch}$.
Le bogie possède une traverse de milieu qui sert d'appui aux :moteurs, ceux-ci tourillonnant, d'autre part, sur l'essieu (suspension par le nez). La caisse est suspendue par pivots renversés, du type "Leboucher ». Cette caisse comporte un compartiment central avec deux cabines de conduite extrême. Les freins sont du type continu à air comprimé Westinghouse, et, en plus, modérables. Il y a également possibilité de commande des freins à la main.

La partie nettement originale de la machine est son équipement 3.000 volts, encore sans équivalent en France. Il comprend quatre moteurs, et un groupe générateur avec batterie pour alimentation des services auxiliaires, puis une excitatrice spéciale, pour la marche en récupération, un équipement de contrôle avec arbre à cames commandés par moteur-pilote pour la marche en traction, et contacteurs électro-magnétiques pour la marche en récupération, les appareils de contrôle et de sécurité.

Pendant la marche en traction, les deux groupes de moteurs sont toujours couplés par deux en série sur un bogie; mais le couplage en série-parallèle est possible pendant la marche en récupération. Les deux groupes série sont reliés en série, chaque moteur fonclionnant en génératrice à action séparée, le courant d'excitation étant réglé à la main en faisant varier le courant d'excitation de l'excitatrice.

Pour assurer la marche stable en régulation, l'excitatrice est munie d'un enroulement de compoundage parcouru par le courant total de récupération. Dans ces conditions, un accroissement du courant débité par la locomotive, dû par exemple ầ une chute de tension en ligne, entrâne une réduction de la tension d'excitatrice, et, par suite, une diminution de la tension Bux bornes de la locomotive et du courant fourni par les machines fonctionnant en génératrice.
Automotrices. - Les C.F.M. ont commandé également à la C.E.F., dix automotrices à 3.000 volts avec quatre moteurs de $175 \mathrm{ch}$, l'un (unihoraire) et $140 \mathrm{ch}$. (continu), dans le but d'effectuer un service de voyageurs rapide $(80-85 \mathrm{~km} . / \mathrm{h}$.$) , destiné$ à soutenir la concurrence avec les autos-cars qui sillonnent, déjà nombreux, les excellentes routes du Maroc. Les deux moteurs de chaque bogie sont normalement couplés en série, sous 1.500 volts. Pour chacun des groupes, possibilité de couplage en série ou en parallèle. La caisse comporte deux cabines de manœuvre aux extrémités, et trois compartiments, dont un fourgon de bagages, un compartiment de première classe à seize places assises, et un compariment de deuxième classe de quarante-huit places assises.

La construction d'automotrices de ce genre est délicate en raison de la haute tension ( 3.000 volts), quand ces motrices travaillent en récupération.

Différentes en ce qui concerne les caisses, ces motrices ont les plus grandes analogies au point de vue équipement avec les automotrices du P.-O. de Paris à Dourdan el Etampes.

On peut constituer, comme avec les précédentes, et suivant l'intensité du tarif des lignes, par exemple sur Casablanca-Rabal, des trafics avec deux remorques et un fourgon (poids total, automotrice comprise : 115 tonnes à vide et 136 tonnes en charge.

Sur la ligne de Casablanca à Kourigha (rampe continue), on ne peut dépasser 85 tonnes à vide et 98 en charge..

Les dimensions caractéristiques de ces automotrices sont les suivantes :

Longueur entre tampons..................

Longueur de la caisse.....................

Largueur de la caisse .....................

Hauteur du toit au-dessus du plan de roulement ...

Empattement rigide d'un bogie ..............

Distance d'axe en axe des bogies................

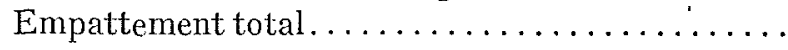

Poids d'un bogie nu .....................

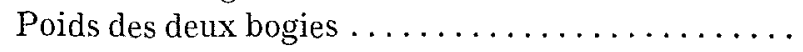

Poids d'un moteur avec engrenages et carter.......

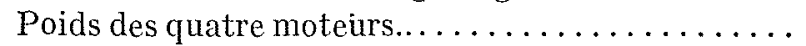

Poids de la caisse nue avec banquettes ..........

Poids de l'équipement de contrôle avec auxiliaire...

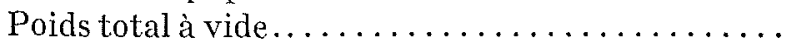

Poids total en charge (environ) ...............

$19 \mathrm{~m} 250$

1820

3

390

3900

11800

14400

8 t. 5

17 tonnes

2 t. 9

11 t. 6

20 t. 7

7 t. 3

66 t. 6

72 tonnes

L'équipement électrique des automotrices comporte :

$1^{\circ}$ Quatre moteurs de traction à 1.500 volts par collecteurs;

$2^{\circ}$ Deux groupes de transformateurs rotatifs à $3.000 / 120$ volts pour alimentation des circuits de contrôle et des compresseurs de frein à air;

\section{$3^{\circ}$ Un équipement de contrôle avec appareils de sécurité;}

$4^{\circ}$ Un appareils auxiliaire pour la captation du courant, compression de l'air, etc...

Les moteurs sont du type "cuirassé " à autoventilation, isolation au mica. Equipement de contrôle avec contacteurs à arbre à cames (fermeture dans un ordre déterminé des deux posilions de connexions de l'équipement).

Et maintenant la conclusion ?

\section{LA CONCLUSION?}

Une entreprise admirable reflétant la confiance et la hardiesse de son grand animateur, M. Parodi, dans la relation éleclrique de la traction de chemins de fer, confiance et hardiesse qu'il a su inspirer à tous les exécutants de ce vaste et si séduisant programme! 